molecular assays in 2020 is interesting. Larger prospective genotypic studies will enable more firm conclusions on emergence of new mutations and/or genotypes in the local population.

Recent studies from Italy and Taiwan revealed disparate findings with regard to AMR prevalence during the COVID-19 pandemic ${ }^{11,12}$ with high rates of AMR attributed in one study to increased antibiotic utilisation in COVID-19 patients. $^{12}$ In contrast to the experience in Europe and Asia, rates of SARS-CoV-2 infection were low in Australia through 2020 and early 2021, so factors relating directly to COVID-19 disease were not at play in this setting. Nonetheless, net antibiotic utilisation data from this study demonstrate a broad trend toward increased use over the period 2015-2021, and we hypothesise this is a major contributor to overall rising rates of invasive ESBL disease.

The observed rise in ESBL invasive disease suggests that local factors are key contributors to MDR Enterobacteriaceae infection in Australia. Alongside net increases in antibiotic use, other contributors may include inappropriate prescribing practices in hospital, community and agricultural settings, or prolonged carriage of MDR organisms in the gastrointestinal tract with invasive disease manifestation months to years later. This study lends weight to the important argument that considered antimicrobial stewardship is as critical in high income settings as it is in low income countries with higher baseline AMR prevalence rates.

AMR remains a critical public health issue faced locally and internationally and is a major focus of public health and research efforts. Interestingly, this study reveals that international travel is likely not the major contributor to the current ESBL epidemic, at least when viewed with a medium term lens. Rather, AMR must be owned as a local health priority driven at least in part by factors such as suboptimal antimicrobial stewardship at a community level. Future large scale studies are welcomed exploring longer term implications of travel restrictions on locally acquired versus internationally acquired ESBL infection rates, as well as ongoing surveillance for emerging mutations or genotypes.

Acknowledgements: The authors are grateful to the staff of the Nepean Hospital microbiology laboratory for their technical assistance and $\mathrm{Mr}$ Robert Robinson for helpful discussions.

Conflicts of interest and sources of funding: The authors state that there are no conflicts of interest to disclose.

Annaleise R. Howard-Jones ${ }^{1,2}$, Samiha Rifaath Anver ${ }^{3}$, Kirsten Tsang ${ }^{4}$, James Branley ${ }^{1,2,3}$

${ }^{1}$ NSW Health Pathology-Nepean, Nepean Hospital, Kingswood, NSW, Australia; ${ }^{2}$ Faculty of Medicine and Health, University of Sydney, Camperdown, NSW, Australia; ${ }^{3}$ Department of Infectious Diseases and Microbiology, Nepean Hospital, Kingswood, NSW, Australia; ${ }^{4}$ Department of Pharmacy, Nepean Hospital, Kingswood, NSW, Australia

Contact Dr James Branley.

E-mail: james.branley@health.nsw.gov.au
1. Bezabih YM, Sabiiti W, Alamneh E, et al. The global prevalence and trend of human intestinal carriage of ESBL-producing Escherichia coli in the community. J Antimicrob Chemother 2021; 76: 22-9.

2. O'Neill J. Tackling Drug-Resistant Infections Globally: Final Report and Recommendations. The Review on Antimicrobial Resistance. May 2016. London: Review on Antimicrobiol Resistance, 2016. https://amrreview.org/sites/default/files/160525_Final\%20paper_with\%20cover. pdf.

3. Broaders E, Gahan CG, Marchesi JR. Mobile genetic elements of the human gastrointestinal tract: potential for spread of antibiotic resistance genes. Gut Microbe 2013; 4: 271-80.

4. Australian Commission on Safety and Quality in Health Care (ACSQHC). AURA 2017: Second Australian Report on Antimicrobial Use and Resistance in Human Health. Sydney: ACSQHC, 2017.

5. Solé M, Pitart C, Oliveira I, et al. Extended spectrum $\beta$-lactamaseproducing Escherichia coli faecal carriage in Spanish travellers returning from tropical and subtropical countries. Clin Microbiol Infect 2014; 20: O636-9.

6. Pires J, Kraemer JG, Kuenzli E, et al. Gut microbiota dynamics in travelers returning from India colonized with extended-spectrum cephalosporin-resistant Enterobacteriaceae: a longitudinal study. Travel Med Infect Dis 2019; 27: 72-80.

7. Australian Bureau of Statistics (ABS). Overseas Arrivals and Departures, Australia (April 2021). Canberra: ABS, 2021.

8. Lee Y, Kim YA, Kim D, et al. Risk factors of community-onset extended-spectrum $\quad \beta$-lactamase-producing Klebsiella pneumoniae bacteraemia in South Korea using national health insurance claims data. Int J Antimicrob Agents 2019; 54: 723-7.

9. Rawat D, Nair D. Extended-spectrum $\beta$-lactamases in Gram negative bacteria. J Glob Infect Dis 2010; 2: 263-74.

10. Birkett CI, Ludlam HA, Woodford N, et al. Real-time TaqMan PCR for rapid detection and typing of genes encoding CTX-M extended-spectrum beta-lactamases. J Med Microbiol 2007; 56: 52-5.

11. Bentivegna E, Luciani M, Arcari L, Santino I, Simmaco M, Martelletti P. Reduction of multidrug-resistant (MDR) bacterial infections during the COVID-19 pandemic: a retrospective study. Int $J$ Environ Res Publ Health 2021; 18: 1003.

12. Lai CC, Chen SY, Ko WC, Hsueh PR. Increased antimicrobial resistance during the COVID-19 pandemic. Int J Antimicrob Agents 2021; 57: 106324 .

DOI: https://doi.org/10.1016/j.pathol.2021.11.005

\section{Identification of Mycobacterium abscessus to subspecies level with Bruker MALDI Biotyper}

To the Editor,

Mycobacterium abscessus is a rapid growing mycobacteria (RGM) which is further separated into three subspecies: Mycobacterium abscessus subsp. abscessus, Mycobacterium abscessus subsp. massiliense, and Mycobacterium abscessus subsp. bolletii. They can cause various pulmonary and nonpulmonary infections, particularly in vulnerable patients with other underlying medical conditions. Macrolides are key antibiotics used in treating these patients, and are given in combination with other companion antimicrobials. ${ }^{1,2}$ Resistance to macrolides correlates with poorer outcomes. The most common mechanism of resistance is due to the presence of functional erm (41) in M. abscessus subsp. abscessus and M. abscessus subsp. bolletii which confers inducible resistance to clarithromycin. ${ }^{3,4}$ Mycobacterium abscessus subsp. massiliense is conversely associated with better outcomes due to truncation of erm (41) rendering it non-functional. Chromosomal mutations in the 23S rRNA ( $r r l)$ gene may result in constitutive macrolide resistance, however this is relatively uncommon. ${ }^{3}$ 
Although RGM such as M. abscessus are culturable within a relatively short time compared to other non-tuberculous mycobacteria, the time required from culture positivity to identification and susceptibility testing requires several weeks. Phenotypic susceptibility testing requires up to 2 weeks incubation time to exclude the presence of inducible clarithromycin resistance as recommended by the Clinical and Laboratory Standards Institute. ${ }^{5}$ While treatment may be delayed for some patients, earlier initiation of treatment may be helpful for patients with particularly serious infections. Rapid identification of $M$. abscessus to the subspecies level thus may be useful to clinicians in determining a suitable empiric antibiotic treatment regimen for these patients prior to availability of full susceptibility testing results. Moreover, it is currently recommended that identification to the subspecies level be performed as repeat positive respiratory samples with the same subspecies is required as part of diagnostic criteria of pulmonary infections. ${ }^{1}$ Thus, we explored the utility of Bruker MALDI Biotyper (Bruker, USA) for identification of M. abscessus to subspecies level.

Between 2017-2019, a collection of 210 Mycobacterium abscessus isolates was fully identified based on whole genome sequencing. ${ }^{6}$ Whole genome sequencing (WGS) was performed on the NovaSeq6000 sequencing platform (Illumina, USA). An average sequencing depth of $150 \times$ was achieved for the genomes. Subspecies identification was determined based on phylogenetic analyses. The MycoEx protocol was used for inactivation and extraction prior to testing on Bruker MALDI Biotyper using the Mycobacteria Library. ${ }^{7}$ Bacterial suspensions were made in $300 \mu \mathrm{L}$ of ultrapure water and inactivated by boiling for $30 \mathrm{~min}$. Cold ethanol $\left(900 \mu \mathrm{L}\right.$ at $\left.-18^{\circ} \mathrm{C}\right)$ was added and mixed thoroughly. The solutions were centrifuged at $13,000 \mathrm{rpm}$ for $2 \mathrm{~min}$ and the supernatant removed. Centrifugation and removal of supernatant was repeated and allowed to air dry. Zirconia/Silica beads (Biospec Products, USA; $0.5 \mathrm{~mm}$ ) and acetonitrile (50 $\mu \mathrm{L}$ ) were added vortexed at maximum speed for $1 \mathrm{~min}$. An equal volume of $70 \%$ formic acid was then added and vortexed at maximum speed for $1 \mathrm{~min}$. Centrifugation at $13,000 \mathrm{rpm}$ for $2 \mathrm{~min}$ was performed and $1 \mu \mathrm{L}$ of supernatant was applied onto the MALDI target plate and allowed to air dry. This was then overlaid with $1 \mu \mathrm{L} \mathrm{MBT} \mathrm{Galaxy} \alpha$-cyano4-hydroxycinnamic acid matrix solution (Bruker) and air dried before loading into the Bruker MALDI Biotyper for testing. The Bruker MALDI Biotyper identifications were compared against the genomic identifications as the reference standard. There were 204 isolates with MALDI score of $\geq 1.800$ included based on previously established cut-offs for high confidence identifications in mycobacteria. ${ }^{7}$ The identification and highest score achieved are stratified based on subspecies identification and MALDI score in Table 1.

Overall, the results indicate that MALDI was not a reliable tool to identify M. abscessus to the subspecies level. There was correct identification in $86.4 \%$ of $M$. abscessus subsp. abscessus and $24.3 \%$ of $M$. abscessus subsp. massiliense. Although correct identification rates were highest in M. abscessus subsp. abscessus, this result was not reliable as $M$. abscessus subsp. massiliense was frequently misidentified as M. abscessus subsp. abscessus (54 M. abscessus subsp. massiliense were misidentified as $M$. abscessus subsp. abscessus). A MALDI result with score of $\geq 1.800$ for M. abscessus subsp. abscessus and M. abscessus subsp. massiliense correctly identified the isolate in 50.9\% (57/112) and $94.3 \%(33 / 35)$ of cases, respectively. This combination of results indicated that a MALDI result of $M$. abscessus subsp. abscessus was relatively sensitive but not specific. Conversely, a MALDI result of $M$. abscessus subsp. massiliense was not sensitive, but relatively specific. A MALDI result for M. abscessus subsp. massiliense was particularly reliable for scores $\geq 2.100$ where no misidentifications

Table 1 Summary of MALDI-TOF results stratified by subspecies identification

\begin{tabular}{|c|c|c|c|c|c|}
\hline \multirow[t]{3}{*}{ Identification by sequencing (no. isolates) } & \multicolumn{5}{|c|}{ MALDI-TOF results } \\
\hline & \multirow{2}{*}{$\begin{array}{l}\text { Isolates with correct } \\
\text { identifications, } n(\%)\end{array}$} & \multirow{2}{*}{$\begin{array}{l}\text { Isolates with incorrect } \\
\text { identifications, } n(\%)\end{array}$} & \multicolumn{3}{|c|}{ Incorrect identifications } \\
\hline & & & $\begin{array}{c}\text { M. abscessus } \\
\text { subsp. abscessus }\end{array}$ & $\begin{array}{c}\text { M. abscessus subsp. } \\
\text { massiliense }\end{array}$ & $\begin{array}{l}\text { M. abscessus } \\
\text { subsp. bolletii }\end{array}$ \\
\hline \multicolumn{6}{|l|}{ MALDI score of $1.800-1.999$} \\
\hline Mycobacterium abscessus subsp. abscessus (18) & $16(88.9 \%)$ & $2(11.1 \%)$ & N/A & 1 & 1 \\
\hline Mycobacterium abscessus subsp. bolletii (1) & $0(0.0 \%)$ & $1(100.0 \%)$ & 1 & 0 & N/A \\
\hline \multicolumn{6}{|l|}{ MALDI score of $2.000-2.099$} \\
\hline Mycobacterium abscessus subsp. abscessus (10) & $9(90.0 \%)$ & $1(10.0 \%)$ & N/A & 1 & 0 \\
\hline Mycobacterium abscessus subsp. massiliense (37) & $7(18.9 \%)$ & $30(81.1 \%)$ & 16 & N/A & 14 \\
\hline Mycobacterium abscessus subsp. bolletii (1) & $1(100.0 \%)$ & $0(0.0 \%)$ & 0 & 0 & N/A \\
\hline \multicolumn{6}{|l|}{ MALDI score of $2.100-2.199$} \\
\hline Mycobacterium abscessus subsp. abscessus (25) & $20(80.0 \%)$ & $5(20.0 \%)$ & N/A & 0 & 5 \\
\hline Mycobacterium abscessus subsp. massiliense (27) & $7(25.9 \%)$ & $20(74.1 \%)$ & 7 & N/A & 13 \\
\hline Mycobacterium abscessus subsp. bolletii (0) & $0(0.0 \%)$ & $0(0.0 \%)$ & 0 & 0 & N/A \\
\hline Mycobacterium abscessus subsp. abscessus (13) & $12(92.3 \%)$ & $1(7.7 \%)$ & N/A & 0 & 1 \\
\hline Mycobacterium abscessus subsp. massiliense (20) & $10(50.0 \%)$ & $10(50.0 \%)$ & 3 & N/A & 7 \\
\hline Mycobacterium abscessus subsp. bolletii $(0)$ & $0(0.0 \%)$ & $0(0.0 \%)$ & 0 & 0 & N/A \\
\hline \multicolumn{6}{|l|}{ All combined (MALDI score $\geq 1.800$ ) } \\
\hline Mycobacterium abscessus subsp. abscessus (66) & $57(86.4 \%)$ & $9(13.6 \%)$ & 0 & 2 & 7 \\
\hline Mycobacterium abscessus subsp. massiliense (136) & $33(24.3 \%)$ & $103(75.7 \%)$ & 54 & 0 & 49 \\
\hline Mycobacterium abscessus subsp. bolletii (2) & $1(50.0 \%)$ & $1(50.0 \%)$ & 1 & 0 & 0 \\
\hline
\end{tabular}


occurred. When a MALDI result of M. abscessus subsp. massiliense with a high score is achieved, the isolate may be confidently identified to subspecies level, which is highly predictive of macrolide susceptibility.

Prior to the MALDI-TOF era, some organisms were proven to be difficult to identify in routine laboratories. The usage of proteomics-based mass spectrometry has revolutionised operations and MALDI is now commonly used for identifying bacteria, fungi, and mycobacteria due to rapidity as well as accuracy. In the context of mycobacteriology, MALDI-TOF can identify commonly encountered NTM species, but is currently unable to identify closely related organisms such as M. abscessus to subspecies level. This is consistent with previous reports. ${ }^{8,9}$ However, we note that a MALDI result of M. abscessus subsp. massiliense is relatively specific, particularly with scores of $\geq 2.100$. Such a result would be highly predictive of the identification being clarithromycin-susceptible $M$. abscessus subsp. massiliense, and may be useful in tailoring empiric antimicrobial therapy particularly for critically ill or immunocompromised patients. Further improvements to the current proteomics-based libraries are required before more reliable subspecies identification can be performed using MALDI-TOF. Modifications to the MALDI approach include lipid-based mass spectrometry. ${ }^{10}$ Initial testing suggests that generated mass spectra can differentiate the M. abscessus subspecies. Although potentially promising, there remains significant validation to be performed with a larger collection of isolates to ensure reproducibility across different laboratories, and this needs to be followed by development of a publicly available database.

Further advances are required for existing MALDI-TOF based technologies before confident identifications to subspecies level can be made. Currently, molecular testing methods remain the best alternative for earlier subspecies identification. This includes polymerase chain reaction testing to identify the presence of truncated or normal length erm (41) genes, PCR and sequencing key genes, or using commercial molecular identification methods.

Conflicts of interest and sources of funding: Whole genome sequencing of the isolates was supported by the National Medical Research Council (NMRC, Singapore) via the Collaborative Solutions Targeting Antimicrobial Resistance Threats in Health System Antimicrobial Resistance Research Grant (CoSTAR-HS/ARGSeedGrant/2019/03). The authors state that there are no conflicts of interest to disclose.

\section{Ka Lip Chew ${ }^{1}$, Patsy Soh ${ }^{1}$, Sophie Octavia ${ }^{2}$, Jeanette Teo $^{1}$}

${ }^{1}$ Department of Laboratory Medicine, National University Hospital, Singapore, Republic of Singapore; ${ }^{2}$ Environmental Health Institute, National Environment Agency, Singapore, Republic of Singapore

Contact Dr Ka Lip Chew.

E-mail: ka_lip_chew@nuhs.edu.sg

1. Daley CL, Iaccarino JM, Lange C, et al. Treatment of nontuberculous mycobacterial pulmonary disease: an official ATS/ERS/ESCMID/IDSA Clinical Practice Guideline. Clin Infect Dis 2020; 71: 1-36.
2. Haworth CS, Banks J, Capstick T, et al. British Thoracic Society guidelines for the management of non-tuberculous mycobacterial pulmonary disease (NTM-PD). Thorax 2017; 72: ii1-64.

3. Chew KL, Cheng JWS, Osman NH, Lin RTP, Teo JWP. Predominance of clarithromycin-susceptible Mycobacterium massiliense subspecies: characterization of the Mycobacterium abscessus complex at a tertiary acute care hospital. J Med Microbiol 2017; 66: 1443-7.

4. Chew KL, Octavia S, Go J. In vitro susceptibility of Mycobacterium abscessus complex and feasibility of standardizing treatment regimens. J Antimicrob Chemother 2021; 76: 973-8.

5. Clinical and Laboratory Standards Institute (CLSI). In: Performance Standards for Susceptibility Testing of Mycobacteria, Nocardia spp. and Other Aerobic Actinomycetes. 1st ed. CLSI supplement M62. Wayne, PA: CLSI, 2018.

6. Chew KL, Octavia S, Jureen R, et al. Molecular epidemiology and phylogenomic analysis of Mycobacterium abscessus complex clinical isolates in an Asian population. Microb Genomics 2021; 7: 000708.

7. Alcaide F, Amlerová J, Bou G, et al. European Study Group on Genomics and Molecular Diagnosis. How to: identify non-tuberculous Mycobacterium species using MALDI-TOF mass spectrometry. Clin Microbiol Infect 2018; 24: 599-603

8. Cao Y, Wang L, Ma P, Fan W, Gu B, Ju S. Accuracy of matrix-assisted laser desorption ionization-time of flight mass spectrometry for identification of mycobacteria: a systematic review and meta-analysis. Sci Rep 2018; 8: 4131

9. Panagea T, Pincus DH, Grogono D, et al. Mycobacterium abscessus complex identification with matrix-assisted laser desorption ionization time of flight mass spectrometry. J Clin Microbiol 2015; 53: 2355-8.

10. Khor MJ, Broda A, Kostrzewa M, Drobniewski F, Larrouy-Maumus G An improved method for rapid detection of Mycobacterium abscessus complex based on species-specific lipid fingerprint by routine MALDITOF. Front Chem 2021; 9: 715890.

DOI: https://doi.org/10.1016/j.pathol.2021.11.014

\section{Cadaveric donor specimens and serological testing for SARS-CoV-2}

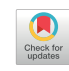

To the Editor,

The coronavirus disease 2019 (COVID-19) pandemic has raised questions around the potential risk of severe acute respiratory syndrome coronavirus 2 (SARS-CoV-2) in tissue and solid organ transplant (SOT) donors and recipients. Cadavers are the major source of donor organs and tissues worldwide. There is a higher risk of severe COVID-19 outcomes related to chronic immunosuppression and associated morbidities. Testing of cadavers for SARS-CoV-2 is limited by the availability and validity of upper respiratory samples, and alternative testing is needed. Screening for SARS-CoV-2 antibodies in donor blood should be given due consideration as a simple and effective way of assessing SARS-CoV-2 infections in cadavers. ${ }^{1}$ Clinical outcomes of tissue and organ recipients from cadaveric donors with prior SARS-CoV-2 infection remain uncertain. Approximately $70 \%$ of organs and tissues used in transplantation are obtained from deceased donors. All of these require screening for blood borne viruses. More recently with the COVID-19 pandemic, issues have arisen around reductions in donors during the pandemic and during times of increased case numbers, and how to deal with the issues of respiratory virus infection in a laboratory setting mainly directed at blood borne pathogens. Retrieval of SOT and tissues from cadavers introduces unique challenges in testing for infectious disease markers, particularly in cases of tissue donation where cadaveric blood is the only source for testing. ${ }^{2}$ Therefore, adequate testing for SARS-CoV-2 antibodies in cadaveric blood is necessary in order to ensure the suitability and safety of the transplantation. 